\title{
Usefulness of double dose contrast-enhanced magnetic resonance imaging for clear delineation of gross tumor volume in stereotactic radiotherapy treatment planning of metastatic brain tumors: a dose comparison study
}

\author{
Kalloo Sharma SUBEDI ${ }^{1 *}$, Takeo TAKAHASHI ${ }^{2}$, Takafumi YAMANO ${ }^{2}$, Jun-ichi SAITOH ${ }^{1}$, \\ Keiichiro NISHIMURA ${ }^{2}$, Yoshiyuki SUZUKI ${ }^{1}$, Tatsuya $\mathrm{OHNO}^{1}$ and Takashi NAKANO ${ }^{1}$
}

${ }^{1}$ Department of Radiation Oncology, Graduate School of Medicine, Gunma University, 3-39-22 Showa-machi, Maebashi, Gunma, 371-8511, Japan

${ }^{2}$ Department of Radiation Oncology, Saitama Medical Center, Saitama Medical University, 1981 Kamoda, Kawagoe, Saitama, 350-8550, Japan

*Corresponding author. Department of Radiation Oncology, Graduate School of Medicine, Gunma University, 3-39-22

Showa-machi, Maebashi, Gunma, 371-8511, Japan. Tel: +81-27-220- 8383 and 8384; Fax: +81-27-220-8397;

Email: drksharma09@gmail.com

(Received 3 April 2012; revised 23 May 2012; accepted 13 June 2012)

\begin{abstract}
The purpose of this study was to compare the size and clearness of gross tumor volumes (GTVs) of metastatic brain tumors on T1-weighted magnetic resonance images between a single dose contrast administration protocol and a double dose contrast administration protocol to determine the optimum dose of contrastenhancement for clear delineation of GTV in stereotactic radiotherapy (SRT). A total of 28 small metastatic brain tumors were evaluated in 13 patients by intra-individual comparison of GTV measurements using single dose and double dose contrast-enhanced thin-slice (1-mm) magnetic resonance imaging (MRI). All patients had confirmed histological types of primary tumors and had undergone hypo-fractionated SRT for metastatic brain tumors. The mean tumor diameter with single dose and double dose contrast-enhancement was $12.0 \pm 1.1 \mathrm{~mm}$ and $13.2 \pm 1.1 \mathrm{~mm}$ respectively $(P<0.001)$. The mean incremental ratio $(\mathrm{MIR})$ obtained by comparing mean tumor diameters was $11.2 \pm 0.02 \%$. The mean volume of GTV-1 (single dose contrastenhancement) and GTV-2 (double dose contrast-enhancement) was $1.38 \pm 0.41 \mathrm{ml}$ and $1.59 \pm 0.45 \mathrm{ml}$ respectively $(P<0.01)$. The MIR by comparing mean tumor volumes was $32.3 \pm 0.4 \%$. The MIR of GTV-1 with $<1 \mathrm{ml}$ volume and GTV-1 with $>1 \mathrm{ml}$ volume was $41.8 \pm 0.05 \%$ and $12.4 \pm 0.03 \%$ respectively $(P<$ 0.001). We conclude that double dose contrast-enhanced thin-slice MRI is a more useful technique than single dose contrast-enhanced thin-slice MRI, especially for clear delineation of GTVs of small metastatic brain tumors in treatment planning of highly precise SRT.
\end{abstract}

Keywords: metastatic brain tumor; contrast enhancement; magnetic resonance imaging; gross tumor volume; stereotactic radiotherapy treatment

\section{INTRODUCTION}

Metastatic brain tumors are the most common types of intracranial tumors. The exact incidence is unknown. The incidence of brain metastasis is increasing, seriously impacting patients' survival and quality of life. This results in a burden to nations and alarming for effective treatment approaches [1-3]. Despite considerable improvement of local control of tumors due to recent advances in radiation therapy, the survival of patients with metastatic brain tumors remains relatively low with a median survival of 612 months, although better local control improves quality of life in some cases [3-6].

Stereotactic radio-surgery (SRS) and hypo-fractionated stereotactic radiotherapy (SRT) are very safe and effective treatment modalities for metastatic brain tumors. SRT has high accuracy in delivery of homogenous high-dose radiation, which is selective to tumor tissue and has a low risk of damage to normal tissue. SRT is widely used in Japan and many other developed countries because of better therapeutic 
results [7-10]. Recent developments in contrast-enhanced magnetic resonance imaging (MRI) technique have proved very useful in detecting even small brain lesions. Due to its higher sensitivity, MRI has become the modality of choice for detection and delineation of brain tumors. Contrast-enhanced MRI is superior to non-enhanced MRI and conventional-dose contrast-enhanced computed tomography (CT) [11-15]. However, there are few reports on the usefulness of double-dose contrast-enhanced MRI in the diagnostic field $[11,12,16]$. To our knowledge, neither any documented data nor research work has yet been reported using this technique for delineation of gross tumor volumes (GTVs) in the field of radiation therapy. GTV is defined as volume of tumor on a T1-weighted contrast-enhanced magnetic resonance (MR) image.

The aim of our study was to compare the size and clearness of GTV of metastatic brain tumors on T1-weighted MR images between a single dose contrast administration protocol and a double dose contrast administration protocol in order to determine the optimum dose of contrast-enhancement for clear delineation of GTV in SRT. Our hypothesis is that double dose contrast-enhanced MRI may be a more useful technique than single dose contrast-enhanced MRI, and may decrease the risk of inaccurate delineation of GTVs of metastatic brain tumors in the treatment planning of highly precise SRT.

\section{MATERIALS AND METHODS}

\section{Study design and subjects of study}

The study design was based on comparison of size and clearness of GTV on single dose and double dose contrast-enhanced T1-weighted MR images of metastatic brain tumors within the same individuals. A total of 28 small metastatic brain tumors in 13 patients were included for evaluation. All patients had confirmed histological types of primary tumors (Table 1). All patients had undergone hypo-fractionated SRT for metastatic brain tumors between November 2006 and April 2011 at our hospitals of Saitama Medical Center, Saitama Medical University and Gunma University. The study was conducted in accordance with the Helsinki Declaration. Informed consent was obtained from all patients.

\section{MRI protocol for imaging of lesions}

MRI was used for the evaluation of GTVs of small metastatic brain tumors before SRT. Gadoteridol (ProHance ${ }^{\circledR}$ ) was used as a contrast medium to enhance metastatic brain tumors in a contrast-enhanced MRI technique. MR images for GTV evaluation before SRT were obtained on a 1.5-T MRI unit (VISART/Progress, Toshiba Medical, Japan) and a 3-T MRI unit (Magnetom Trio A Tim, Siemens, Germany). The single dose contrast-enhancement (GTV-1) protocol utilized a dose of gadoteridol $(0.1 \mathrm{mmol} / \mathrm{kg})$ injected intravenously. T1-weighted axial images were obtained three minutes after injection by the fast-spin echo method with 1-mm slice thickness of MR image without a gap. For the double dose contrast-enhancement (GTV-2) protocol a second identical dose of gadoteridol was administered intravenously. Following administration of the second contrast dose, identical T1-weighted images were acquired in the same planes, angulations and positions. No adverse effect of contrast medium was observed.

\section{Image analysis and GTV delineation}

The T1-weighted MR images obtained from each patient following both the single dose and the double dose contrast-enhanced protocols were transferred to the online SRT treatment planning system (iPlan, Brain LAB). This planning system automatically reconstructs the volumetric images of metastatic brain tumors. These images were delineated by experienced radiation oncologists who were blind to the information of contrast medium and dose administration. All images were manually outlined slice by slice, and carefully analyzed in descriptive terms including diameters, volumes, enhancement of lesions and clearness of contour delineation. GTV measurement consisted of enhanced gross tumor tissue, and clinical target volume (CTV) consisted of GTV with an additional margin of about $2 \mathrm{~mm}$. GTV was determined on T1-weighted contrast-enhanced MR images obtained following single dose and double dose contrast administration.

\section{Statistical analysis}

Data were analyzed using Microsoft Office Excel 2007 and commercial statistical software (GraphPad Prism, version 5.0 for Windows, GraphPad Prism Software, San Diego, CA, USA). A two-tailed paired T-test was used to evaluate statistical significance of differences between tumor measurements (diameter and GTV) obtained following single dose and double dose contrast-enhancement. A nonparametric Mann Whitney (two-tailed) test was also used to compare MIR between GTV-1 with $<1 \mathrm{ml}$ volume and GTV-1 with $>1 \mathrm{ml}$ volume. MIR, or mean incremental ratio, is defined as the mean ratio of additional increase of mean tumor measurement (as a \%) obtained after doubledose contrast-enhanced MRI in relation to those measurements obtained after single dose contrast-enhanced MRI. MIR was calculated using the equation:

$$
\mathrm{MIR}=\left(\frac{\mathrm{GTV}-2-\mathrm{GTV}-1}{\mathrm{GTV}-1}\right) \times 100[\%]
$$

A $P$ value $<0.05$ was considered to be significant. Data were expressed as mean \pm standard error. 


\section{RESULTS}

\section{Characteristics of patients and lesions}

Characteristics of patients and lesions are shown in Table 1. Of the 13 patients, nine were male and four female. Age ranged from 45 to 66 years old with a median of 56 years old. Eleven patients had lung cancer, one had breast cancer and one had ovarian cancer. By histology, 12 patients had adenocarcinoma and one patient had squamous cell carcinoma. Lung cancer was the primary site for $85.7 \%$ of metastatic brain tumors. By histology, $96.4 \%$ of lesions were adenocarcinomas. According to the location of metastatic brain tumors, $28.5 \%$ of lesions were in the parietal lobe, $21.4 \%$ of lesions in the temporal lobe, $21.4 \%$ of lesions in the frontal lobe, $10.7 \%$ of lesions in the occipital lobe and $10.7 \%$ of lesions in the cerebellum. Some $(7.3 \%)$ of lesions were in the thalamus and corpus callosum as well.

\section{Measurements and evaluation of lesions}

Diameters of metastatic brain tumors obtained by singledose contrast-enhanced MRI ranged from 4.0-28.0 mm with a mean of $12.0 \pm 1.1 \mathrm{~mm}$, and those by double dose contrast-enhanced MRI ranged from 4.4-29.0 mm with a mean of $13.2 \pm 1.1 \mathrm{~mm}(P<0.001)$. The MIR by comparing mean tumor diameters was $11.2 \pm 0.02 \%$. Such a small

Table 1. Characteristics of patients and lesions:

\begin{tabular}{lccc}
\hline Characteristics & $\begin{array}{c}\text { No. of } \\
\text { Patients }(\%)^{\text {a }}\end{array}$ & $\begin{array}{c}\text { Lesions } \\
\text { No. }(\%)\end{array}$ & $\begin{array}{c}\text { MIR } \\
(\%)\end{array}$ \\
\hline Total & $\mathbf{1 3}$ & $\mathbf{2 8}$ & $\mathbf{3 2 . 3}$ \\
Male & $9(69.2)$ & $19(67.8)$ & \\
Female & $4(30.8)$ & $9(32.2)$ & \\
Primary lesions: & & & \\
Lung Cancer & $11(84.6)$ & $24(85.7)$ & 35 \\
Breast cancer & $1(7.7)$ & $3(10.7)$ & 19.7 \\
Ovarian Cancer & $1(7.7)$ & $1(3.6)$ & 5.7 \\
Histology type: & & & \\
Adenocarcinoma & $12(92.3)$ & $27(96.4)$ & 33.3 \\
Sq. cell carcinoma & $1(7.7)$ & $1(3.6)$ & 6.9 \\
Classification & & & \\
Group 1 $(<1 \mathrm{ml})$ & $19(67.8)$ & 41.8 & \\
Group 2 $(1-3 \mathrm{ml})$ & $6(21.4)$ & 12.6 & \\
Group 3 $(>3 \mathrm{ml})$ & $3(10.7)$ & 12.0 & \\
(Lesions with $>1 \mathrm{ml})$ & $9(32.1)$ & 12.4 & \\
\hline
\end{tabular}

${ }^{\text {a}}$ Patient's age ranged from 45 to 66 years with a mean of 56 years.

${ }^{\mathrm{b}}$ Based on a single dose contrast-enhanced volume. MIR = mean incremental ratio. Sq. $=$ squamous. incremental ratio may not be valuable in the clinical practice of SRT planning. The mean of GTV-1 was $1.38 \pm$ $0.41 \mathrm{ml}$, and the mean of GTV-2 was $1.59 \pm 0.45 \mathrm{ml}(P<$ 0.01). The MIR obtained by comparing the mean of GTV-1 and GTV-2 was $32.3 \pm 0.4 \%$, which is valuable and useful in the clinical practice of SRT planning. GTV-2 was more clearly visualized and prominently larger than GTV-1 in all cases (Fig. 1A and Fig. 2).

It was also observed that $21.4 \%$ of lesions demonstrated more than $50 \%$ increment $(\mathrm{MIR}=66.9 \pm 6.0 \%)$ in volume, $32.1 \%$ of lesions showed $25-50 \%$ increment (MIR $=34.4 \pm$ $2.6 \%$ ) in volume and $46.4 \%$ of lesions showed less than $25 \%$ increment $(\mathrm{MIR}=14.9 \pm 2.2 \%)$ in volume. Based on tumor volumes obtained by a single dose contrast-enhancement, we classified lesions in three groups as shown in Table 1. By this classification, $67.8 \%$ of lesions with $<1 \mathrm{ml}$ volume were classified in group 1 with MIR of $41.8 \pm 0.05 \% ; 21.4 \%$ of lesions with $1-3 \mathrm{ml}$ volume were classified in group 2 with MIR of $12.6 \pm 3.4 \% ; 10.7 \%$ of lesions with $>3 \mathrm{ml}$ volume were classified in group 3 with MIR of $12.0 \pm 5.7 \%$. Similarly, $32.1 \%$ of lesions with $>1$ $\mathrm{ml}$ volume showed MIR of $12.4 \pm 0.03 \%$. Lesions with $<1$ $\mathrm{ml}$ volume were more clearly visualized with prominently larger volume and significantly greater MIR than those with $>1 \mathrm{ml}$ volume $(P<0.001)$ as shown in Fig. $1 \mathrm{~B}$ and Fig. 2. This is an important result of the present study, which may be useful for SRT planning, especially for small metastatic brain tumors with $<1 \mathrm{ml}$ volume in size.

\section{DISCUSSION}

Metastatic brain tumors may originate from any primary site. The most common sites of origin for metastatic brain
A

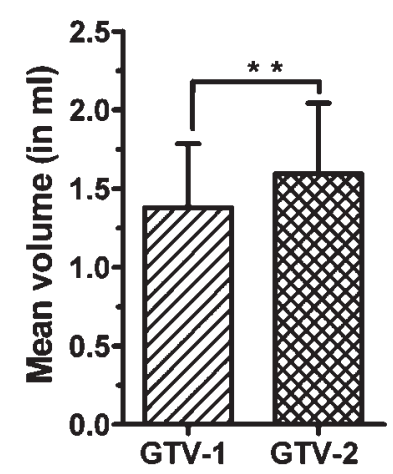

B

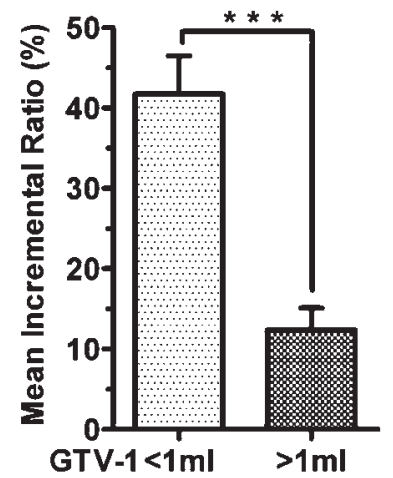

Figure 1: Comparison of mean gross tumor volumes (GTVs) and mean incremental ratio (MIR) of metastatic brain tumors. (A) Mean volume of GTV-2 (double dose contrast-enhancement) is demonstrated to be prominently larger than GTV-1 (single dose contrast-enhancement). (B) MIR of GTV-1 with $<1 \mathrm{ml}$ volume is demonstrated to be prominently greater than GTV-1 with $>1 \mathrm{ml}$ volume. Error bar represents standard error. 


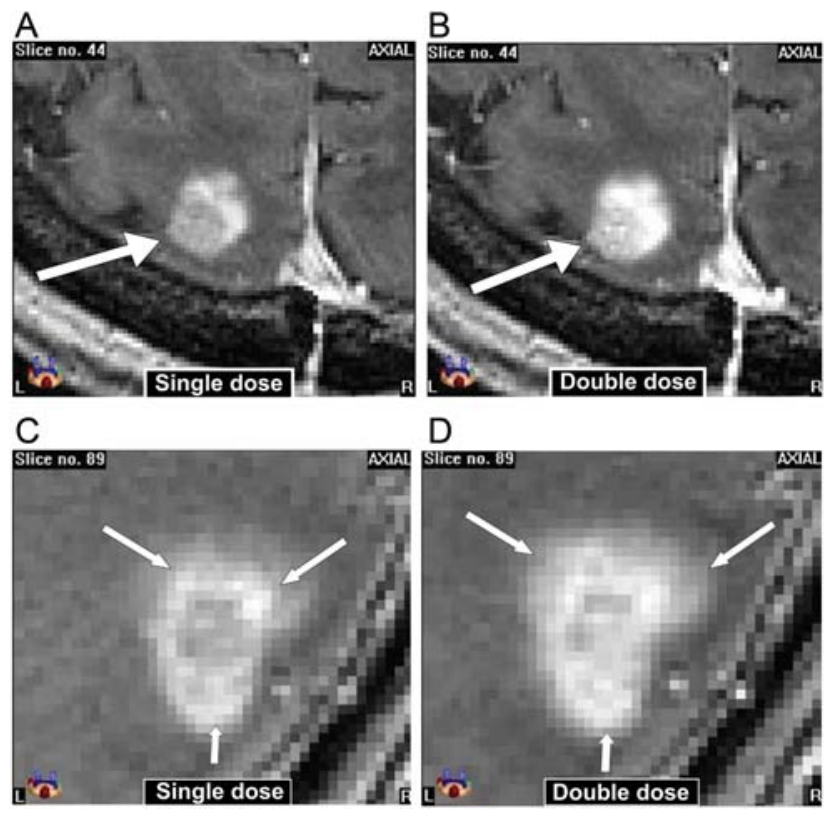

Figure 2: Comparison of MR images of metastatic brain tumors between single dose and double dose contrast-enhancement. T1-weighted MR images (B) and (D) (double dose contrast-enhancement) demonstrate clearer visualization with prominently larger volume, better enhancement and clearer margin than (A) and (C) (single dose contrast-enhancement) images. Enhanced tumors are shown by white arrows.

tumors are lung, breast, colon and rectum [1]. In our study, the majority $(85.7 \%)$ of metastatic brain tumors originated from lung cancer (Table 1), and the parietal lobe was the most common site of metastasis. The majority $(67.8 \%)$ of lesions were $<1 \mathrm{ml}$ in volume (Table 1 ) and had an irregular shape. Assessment of these small lesions by comparing GTV demonstrated a significant difference between singledose and double dose contrast-enhanced MRI (Fig. 1A).

Accurate delineation of GTV is essential in proper dose planning for successful SRT. Accurate GTV delineation depends on use of higher doses of contrast medium (as demonstrated in this study), as well as thinner image slices through the tumor and accurate positioning of the fixation device attached to the linear accelerator [17-19]. Assessment of tumor targeting with accurate delineation of GTV is a very challenging task because the majority of metastatic brain tumors occur by hematogenous spread of cancer cells, which proliferate and extend to the surrounding brain tissue irregularly by a neovascularization process. The most important and difficult factor is that presently no imaging modality can detect a total entire extension of tumor with surrounding microscopic spread. Although the optimum dose of contrast medium for contrast-enhanced MRI in the treatment planning of SRT has not yet been determined, in the conventional method of GTV delineation for SRT a single dose of contrast medium for MRI is used to enhance metastatic brain tumors. In our study, we used both a single dose and double dose contrast-enhanced MRI to determine the optimum dose of contrast-enhancement. The present study clearly demonstrates that double dose contrast-enhanced MRI is a more useful technique than single dose contrast-enhanced MRI for clear delineation of GTV in SRT.

The paramagnetic contrast medium does not penetrate the intact blood-brain barrier (BBB). Hence, contrast medium is not accumulated in normal brain tissue with a normal $\mathrm{BBB}$, whereas neoplastic lesions with disruption of the $\mathrm{BBB}$, or abnormal vascularity, allow accumulation of contrast medium. Single dose contrast-enhancement may not precisely encompass the total area of tumor tissue and lesions may appear smaller than actual size with some hazy and punctate patterns of enhancement. This may cause an inaccurate GTV delineation, which may lead to inappropriate treatment dose planning to the targeted tumor in SRT. However with double dose contrast-enhancement the amount of contrast medium encompasses a wider area, which permits a clearer visualization of the BBB breakdown area including the surrounding area of tumor infiltration. In this study, GTV-2 were larger with clearer margins and better enhancement than GTV-1 (Fig. 2). Lesions $<1$ $\mathrm{ml}$ in volume were more clearly visualized with a prominently larger volume and significantly greater MIR than lesions $>1 \mathrm{ml}$ in volume (Fig. 1B and Fig. 2). This may facilitate a clearer delineation of GTV, especially of small metastatic brain tumors for appropriate treatment dose planning in SRT (Fig. 3).

Engelhorn et al. showed the accuracy of double dose contrast-enhanced MRI and contrast-enhanced CT techniques in vivo in a rat model with malignant glioma. A good correlation was observed between double dose contrast-enhanced MRI and micro-CT-derived tumor volumes with histology [20]. To our knowledge a similar study of human patients undergoing clinical radiotherapy
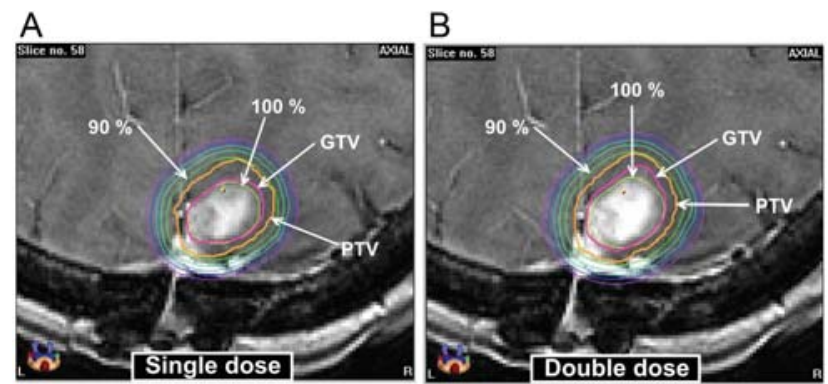

Figure 3: Comparison of dose distribution of SRT on gross tumor volume (GTV) of metastatic brain tumors. T1-weighted MR images demonstrate that GTV-2 (double dose contrast-enhancement) in image (B) is clearer and larger than GTV-1 (single dose contrastenhancement) in image (A). The dose distribution planning of SRT made by GTV-1 delineation is not enough to encompass GTV-2 more precisely. Dose distribution planning is shown by white arrows. 
has not been previously reported. Engelhorn's study supports the finding that the GTV of metastatic brain tumors with double dose contrast-enhanced MRI is larger and more clearly visualized than that with single dose contrast-enhanced MRI.

There were some limitations in our study. First, we were unable to obtain a large number of patients or lesions in the limited period of our prospective study. Second, a repeat MRI with an additional dose of contrast medium was not performed in all patients considered for inclusion in our study. However, the data of our study was statistically significant in demonstrating the usefulness of the double dose contrast-enhanced MRI technique in clear delineation of GTVs for SRT of metastatic brain tumors.

\section{CONCLUSION}

Our study demonstrates the usefulness of a double dose contrast-enhanced MRI protocol compared to a similar single dose contrast-enhanced MRI protocol, especially for clear delineation of GTVs of small metastatic brain tumors. This may be used as a clinically valid and useful technique in highly precise SRT expecting more clinical applications in future.

\section{ACKNOWLEDGEMENTS}

We gratefully thank the International Atomic Energy Agency (IAEA), Hara hospital (Gunma, Japan) and the Government of Nepal for providing partial support and recommendation for K.S. Subedi to continue his Ph.D. fellowship in Radiation Oncology. The above agencies had no role in study design, data collection or manuscript preparation for this study. We also thank Dr William E. Brant, (Prof. Emeritus, Department of Radiology, University of Virginia, USA) for his helpful effort in reviewing this manuscript for linguistic style and grammatical corrections, and providing valuable comments and suggestions.

\section{REFERENCES}

1. Gavrilovic I, Posner J. Brain metastases: epidemiology and pathophysiology. J Neurooncol 2005;75:5-14.

2. Jemal A, Siegel R, Xu J et al. Cancer Statistics, 2010. CA Cancer J Clin 2010;60:277-300.

3. Lohr F, Pirzkall A, Hog $\mathrm{H}$ et al. Adjuvant treatment of brain metastases. Semin Surg Oncol 2001;20:50-6.

4. Andrews DW, Scott CB, Sperduto PW et al. Whole brain radiation therapy with or without stereotactic radiosurgery boost for patients with one to three brain metastases: Phase III results of the RTOG 9508 randomized trial. Lancet 2004;363:1665-72.

5. Ranjan T, Abrey L. Current management of metastatic brain disease. Neurotherapeutics 2009;6:598-603.
6. Saitoh J, Saito Y, Kazumoto T et al. Therapeutic effect of linac-based stereotactic radiotherapy with a micro-multileaf collimator for the treatment of patients with brain metastases from lung cancer. Jpn J Clin Oncol 2010;40:119-24.

7. Aoyama H, Shirato H, Onimaru R et al. Hypofractionated stereotactic radiotherapy alone without whole-brain irradiation for patients with solitary and oligo brain metastasis using noninvasive fixation of the skull. Int J Radiat Oncol Biol Phys 2003;56:793-800.

8. Shaw E, Scott C, Souhami L et al. Single-dose radiosurgical treatment of recurrent previously irradiated primary brain tumors and brain metastases: final report of RTOG protocol 90-05. Int J Radiat Oncol Biol Phys 2000;47:291-8.

9. Tokuuye K, Akine Y, Sumi M et al. Fractionated stereotactic radiotherapy of small intracranial malignancies. Int $J$ Radiat Oncol Biol Phys 1998;42:989-94.

10. Williams JA. Fractionated stereotactic radiotherapy for acoustic neuromas. Int J Radiat Oncol Biol Phys 2002;54:500-4.

11. Aoyama $\mathrm{H}$, Shirato $\mathrm{H}$, Nishioka $\mathrm{T}$ et al. Magnetic resonance imaging system for three-dimensional conformal radiotherapy and its impact on gross tumor volume delineation of central nervous system tumors. Int $J$ Radiat Oncol Biol Phys 2001;50:821-7.

12. Brekenfeld C, Foert E, Hundt W et al. Enhancement of cerebral diseases: how much contrast agent is enough? Comparison of $0.1,0.2$, and $0.3 \mathrm{mmol} / \mathrm{kg}$ gadoteridol at 0.2 $\mathrm{T}$ with $0.1 \mathrm{mmol} / \mathrm{kg}$ gadoteridol at $1.5 \mathrm{~T}$. Invest Radiol 2001;36:266-75.

13. Kim ES, Chang JH, Choi HS et al. Diagnostic yield of double-dose gadobutrol in the detection of brain metastasis: intraindividual comparison with double-dose gadopentetate dimeglumine. AJNR Am J Neuroradiol 2010;31:1055-8.

14. Schellinger PD, Meinck HM, Thron A. Diagnostic accuracy of MRI compared to CECT in patients with brain metastases. J Neurooncol 1999;44:275-81.

15. Suzuki K, Yamamoto M, Hasegawa Y et al. Magnetic resonance imaging and computed tomography in the diagnoses of brain metastases of lung cancer. Lung Cancer 2004;46:357-60.

16. Tatsuno S, Hata Y, Tada S. Double-dose GD-DTPA: detectability of intraparenchymal brain metastasis. Nihon Igaku Hoshasen Gakkai Zasshi 1996;56(12):855-9 (in Japanese).

17. Engh JA, Flickinger JC, Niranjan A et al. Optimizing intracranial metastasis detection for stereotactic radiosurgery. Stereotact Funct Neurosurg 2007;85:162-68.

18. Yuh WT, Tali ET, Nguyen HD et al. The effect of contrast dose, imaging time, and lesion size in the MR detection of intracerebral metastasis. AJNR Am $J$ Neuroradiol 1995;16:373-80. [Published erratum appears in AJNR Am J Neuroradiol 1995;16:1384.]

19. Takahashi T, Nishimura K, Hondo $\mathrm{M}$ et al. Measurement of repositioning accuracy during fractionated stereotactic radiotherapy for intracranial tumors using noninvasive fixation of BrainLAB radiotherapy equipment. Int J Radiat Oncol Biol Phys 2006;66:S67-S70.

20. Engelhorn T, Eyupoglu IY, Schwarz MA et al. In vivo micro-CT imaging of rat brain glioma: a comparison with $3 \mathrm{~T}$ MRI and histology. Neurosci Lett 2009;458:28-31. 